\title{
Age-adjusted charlson comorbidity index and 30-day morbidity in pelvic surgeries
}

\author{
Sampada B. Dessai, R. Fasal, J. Dipin, D.Adarsh, Satheesan Balasubramanian
}

\begin{abstract}
Introduction: Charlson comorbidity index $(\mathrm{CCl})$ is a validated tool enabling clinicians for prediction of adverse events posttherapy. In this study, we planned to estimate the predictive value of age-adjusted $\mathrm{CCl}(\mathrm{ACCl})$ in assessing the perioperative complication in oncological patients undergoing major pelvic surgeries. Methods: This was a single arm, prospective, observational study, in which adult patients with pelvic malignancies undergoing pelvic surgeries were selected. The relationship between the ACCl and Grade 3-5 adverse events were tested using Fisher's test. Results: The rate of Grade 3-5 adverse event rate was $16.7 \%$ (I I patients, $n=66$ ).Among the whole cohort, I I patients ( $16.7 \%$ ) had high score on ACCI.The rate of Grade 3-5 adverse events was higher in the cohort of patients with high ACCl score (45.5\% vs. $10.9 \%, P=0.014)$. The sensitivity, specificity and negative and positive predictive values were $45.5 \%, 89.1 \%, 89.1 \%$, and $45.5 \%$, respectively. Conclusion: $\mathrm{ACCl}$ can predict for postsurgical adverse events. It has a high negative predictive value for nonoccurrence of adverse events.

Key words:Adverse events, age adjusted Charlson comorbidity index, Charlson comorbidity index, complications, pelvic, surgeries
\end{abstract}

\section{Introduction}

Cancer is the disease of the elderly. ${ }^{[1]}$ Frequently cancer patients have concomitant comorbidities. ${ }^{[2]}$ These comorbidities have a negative impact on the prognosis of these patients. ${ }^{[2]}$ Comorbidities lead to an increased incidence of postoperative morbidity, increase likelihood of delay, and incompletion of adjuvant therapy (radiation and or chemotherapy). ${ }^{[3]}$ These comorbidities lead to a higher incidence of both cancer-related and cancer unrelated deaths.

Surgery remains the mainstay of curative treatment in the majority of solid tumors. Deep surgeries, especially coelomic cavity surgeries are associated with high chances of postoperative morbidity. Pelvic cavity surgeries, especially done for epithelial ovarian cancer, rectal cancer, endometrial cancer, uterine cervical cancer, and urinary bladder malignancy are associated with higher incidence of postoperative adverse events. Unfortunately, some of these malignancies such as epithelial ovarian cancer, rectal cancer, and endometrial cancer are commonly associated with medical comorbidities too. ${ }^{[4-6]}$ Prediction of postoperative adverse events would require unable surgeons to better plan therapy in such patients.

Charlson comorbidity index (CCI) is a validated tool enabling clinicians for prediction of adverse events posttherapy. ${ }^{[7]}$ Age is an independent prognostic factor for prediction of adverse events post therapy and is subsequently incorporated in CCI as age-adjusted CCI (ACCI) ${ }^{[8]}$ Both these tools are validated in the Western world and data regarding its validation from India is missing. We work at a tertiary cancer center located in rural India; allocation of appropriate resource is an important priority for us. In this study, we planned to estimate the predictive value of ACCI in assessing the perioperative complication in oncological patients undergoing major pelvic surgeries.

\section{Methods}

\section{Study design and conduct}

This was an Institutional Review Board approved, single arm, single center, prospective observational study. The study protocol was not amended post-approval. The study was conducted by good clinical practice guidelines and declaration

\begin{tabular}{|c|}
\hline Access this article online \\
\hline 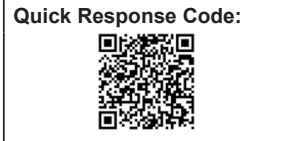 \\
\hline Website: www.sajc.org \\
\hline DOI: 10.4103/sajc.sajc_241_17 \\
\hline
\end{tabular}

of Helsinki. All patients provided written informed consent before enrollment in the study.

\section{Participants}

Adult patients with either radiological or pathological proof of malignancy, with the Eastern Cooperative Oncology Group Performance Status 0-2, undergoing major pelvic surgery with curative intent were included for this study. Patients who underwent surgery for recurrent cancer were excluded from this study.

\section{Setting}

The study was conducted in the principal investigator's institute in the Department of Surgical Oncology between October 2015 and July 2016. Patients subjected to above mention selection criteria were invited to participate in the study. Post-enrollment in the study, CCI and ACCI were assessed. Two clinicians independently assessed the indices. Any discrepancy in finding between the two clinicians was addressed via discussion. No change in surgical or postsurgical plan was contemplated on the basis of scores of CCI and ACCI. These patients then underwent the workup for diagnosis and staging of the respective malignancy in accordance with the National comprehensive cancer network guidelines. Intraoperative, perioperative, and postoperative adverse events until day 30 postsurgery were recorded.

\section{Variables and data measurement}

Data of comorbidity before the diagnosis of cancer and at hospital admission for surgery were collected, and CCI and ACCI were calculated. Baseline data of patients' age, gender, comorbidity, body mass index, primary disease site, stage of disease preoperative albumin, the presence of ascites was recorded and prior neoadjuvant chemotherapy (NACT)/NACT real-time data were collected.

Intraoperative details such as the extent of resection, need for upper abdominal procedures, blood loss, any intraoperative complications, and operative time were recorded. Complications were graded according to CTCAE 4.02 version.

This is an open access journal, and articles are distributed under the terms of the Creative Commons Attribution-NonCommercial-ShareAlike 4.0 License, which allows others to remix, tweak, and build upon the work non-commercially, as long as appropriate credit is given and the new creations are licensed under the identical terms.

Center, Kannur, Kerala, India

Correspondence to: Dr. Sampada B. Dessai, E-mail:sampadads@gmail.com
For reprints contact: reprints@ @medknow.com

How to cite this article: Dessai SB, Fasal R, Dipin J, Adarsh D, Balasubramanian S. Age-adjusted charlson comorbidity index and 30-day morbidity in pelvic surgeries. South Asian J Cancer 2018;7:240-3. 


\section{Study size}

The sample size of 200 surgeries was calculated with the assumption of complication rate of $50 \%$, with an alpha of $5 \%$, with an estimated sensitivity of $85 \%$, with a marginal error rate estimate of $7 \%$ with $95 \%$ confidence interval. ${ }^{[9]}$ However, the study had to be culminated after accrual of 66 cases due to slow recruitment.

\section{Quantitative variables}

1. ACCI calculation was done in accordance with the methodology provided below. In accordance with [Table 1], for each comorbidity, a score was given. Then total score was obtained by adding the score given for each comorbidity. To this score age adjustment was done, i.e., for each decade after 40 years, add 1 point to total score (i.e., 1 point for age group 50-59 years, 2 points for age group 60-69

2. Adverse event rate - Adverse events during surgery and occurring within 30 days of surgery were noted. These

Table 1: Scoring used for calculation of age-adjusted Charlson comorbidity index

\begin{tabular}{ll}
\hline Score & Comorbidity \\
\hline 1 & Diabetes mellitus without end-organ damage \\
& Cerebrovascular disease \\
& Myocardial infarction \\
& Congestive heart failure \\
& Peripheral vascular disease \\
& Dementia \\
& Chronic pulmonary disease \\
& Connective tissue disease \\
& Peptic ulcer disease \\
& Mild liver disease \\
& Diabetes mellitus with end-organ damage \\
& Moderate/severe renal disease \\
& Hemiplegia \\
& Solid tumor without metastasis \\
& (exclude if $>5$ years from diagnosis) leukemia \\
& Lymphoma \\
& Moderate/severe liver disease \\
& Metastatic solid tumor \\
& AIDS (not just HIV positive) \\
\hline
\end{tabular}

adverse events were graded by CTCAE version 4.03

3. Length of hospitalization (LH) - The length of postoperative hospitalization was calculated in days. LH was calculated from the date of surgery until the date of discharge. In case if the patient required readmission for toxicity, then the length of readmission was included in the calculation of LH.

\section{Statistical methods}

SPSS version 16 (Chicago, SPSS Inc) and $R$ version 3.3.0 (Vienna, Austria) was used for analysis. The ACCI $\geq 6$ was considered as high risk of adverse events and a score below 6 was considered as low risk of adverse events. The relationship between ACCI score and occurrence of any grade adverse event/grade 3-5 adverse event was tested using Fisher's test. A value of $P=0.05$ was considered as statistically significant. The impact of ACCI was tested on multivariate analysis by using binary logistic regression analysis. The dependent variable was the occurrence of adverse events. The independent variable tested were ACCI cohort ( $\geq 6$ vs. $<6$ ), tumor site (ovary vs. non ovary), and previous treatment of chemotherapy or radiation (Yes/No). Adjusted odds ratio was calculated for the occurrence of an adverse event in ACCI $\geq 6$ score cohort. A $P=0.05$ was taken as statistically significant. The sensitivity, specificity, and positive and negative predictive value of ACCI for prediction of occurrence of any grade and Grade 3-5 adverse events was calculated. The LH between the two cohorts of ACCI was compared using unpaired $t$-test. A $P=0.05$ was considered as statistically significant.

\section{Results}

\section{Baseline details}

Sixty-six cases were recruited. The baseline details are shown in Table 2. The median age of the whole cohort was 56.5 years ( Interquartile range (IOR) 48.7-56.5). There was a female preponderance as we had 54 females $(83.1 \%)$. The most common comorbidities seen were hypertension in 22 patients $(33.8 \%)$, diabetes mellitus in 14 patients $(21.5 \%)$,

Table 2: Baseline details

\begin{tabular}{|c|c|c|c|}
\hline Variable & ACI $<6(n=55)$ & $\mathrm{ACI} \geq 6(n=11)$ & Total $(n=66)$ \\
\hline Mean age (years) & 55.0 (range $24-82$ ) & 55.5 (range $42-72$ ) & 55.5 (range 24-82) \\
\hline \multicolumn{4}{|l|}{ Gender, $n(\%)$} \\
\hline Female & $46(83.6)$ & $10(90.9)$ & $54(81.8)$ \\
\hline Male & $9(16.4)$ & $1(9.1)$ & $12(17.2)$ \\
\hline Median BMI $\left(\mathrm{kg} / \mathrm{m}^{2}\right)$ & 34.4 (range 21.2-47.0) & 33.2 (range 22.4-44.9) & 33.9 (range 21.2-44.9) \\
\hline \multicolumn{4}{|l|}{ Site of malignancy, $n(\%)$} \\
\hline Ovary & $18(32.7)$ & $9(81.8)$ & $27(41.5)$ \\
\hline Endometrium & $14(25.5)$ & $1(9.1)$ & $15(23.1)$ \\
\hline Uterine cervix & $7(12.7)$ & $1(9.1)$ & $8(12.3)$ \\
\hline Rectum/sigmoid/colon & $14(25.5)$ & - & $14(21.2)$ \\
\hline Urinary bladder & $2(3.6)$ & - & $2(1.9)$ \\
\hline \multicolumn{4}{|l|}{ Comorbidity, $n(\%)$} \\
\hline Hypertension & $20(36.4)$ & $2(18.2)$ & $22(33.8)$ \\
\hline Diabetes mellitus & $11(20.0)$ & $3(27.3)$ & $14(21.5)$ \\
\hline COPD & $7(12.7)$ & $1(9.1)$ & $8(12.3)$ \\
\hline IHD & $1(1.4)$ & $2(18.2)$ & $3(4.6)$ \\
\hline \multicolumn{4}{|l|}{ Previous treatment } \\
\hline Neoadjuvant chemotherapy & $11(20.0)$ & $9(81.8)$ & $20(30.8)$ \\
\hline Neoadjuvant chemoradiation & $6(11.3)$ & $1(9.1)$ & $7(10.8)$ \\
\hline
\end{tabular}

$\mathrm{ACI}=$ Age-adjusted Charlson comorbidity index, $\mathrm{COPD}=$ Chronic pulmonary disease, $\mathrm{IHD}=$ Ischemic heart disease, $\mathrm{BMI}=$ Body mass index 
chronic obstructive pulmonary disease in 8 patients $(12.3 \%)$, and ischemic heart disease in 3 patients $(4.6 \%)$. The commonest site of primary was ovary in 27 patients $(41.5 \%)$, endometrium in 15 patients $(23.1 \%)$, and rectum or colon in 14 patients $(21.2 \%)$.

\section{Treatment details}

Twenty-seven patients (41.6\%) had received previous therapy. Twenty patients with ovarian malignancy had received NACT before surgery. The NACT consisted of 3 cycles of 3 weekly paclitaxel and carboplatin. Seven patients had received preoperative chemoradiation.

The details of surgical resection are shown in Table 3. Pelvic lymph node dissection was done in 60 cases of 66 . Reexploration was done in 3 patients. The median postoperative admission was for 7.0 days (interquartile range 5-10 days).

Adverse event and adjusted Charlson comorbidity index prediction

The adverse event rate is shown in Table 4 . The rate of Grade 3-5 adverse event rate was $16.7 \%$ (11 patients, $n=66)$. Among the whole cohort, 11 patients (16.7\%) had a high score on CCI and ACCI. The rate of Grade 3-5 adverse events was higher in the cohort of patients with high CCI score $(41.7 \%$ vs. $11.1 \%$, p-0.022) [Table 5] and high ACCI score $(45.5 \%$ vs. $10.9 \%$, p-0.014) Table 6. On multivariate analysis, ACCI score showed a statistical trend towards prediction of occurrence of adverse events Table 7. The sensitivity, specificity, and negative and positive predictive values were $45.5 \%, 89.1 \%, 89.1 \%$, and $45.5 \%$, respectively.

The mean length of postoperative stay in a cohort of patients with low ACCI score was 7.7 days (standard deviation [SD] 4.5 days) while that in patients with high ACCI score was 9.4 days (SD 4.8 days) $(P=0.319)$.

\section{Discussion}

This study confirmed the importance of CCI and ACCI in patients undergoing pelvic surgeries for pelvic malignancies. Among a cohort of patients having a 6 or more score on ACCI, the proportion of grade 3-5 adverse events was $45.5 \%$ against $10.9 \%$ in patients with a score $<6$. Similar prediction was seen with CCI score of 4 or more. Both CCI and ACCI were defined for prediction of perioperative complications and overall survival postsurgery. ${ }^{[7]}$ Subsequently, these scores have been validated in a number of populations and for prediction of complications to other treatment modalities too. ${ }^{[10-13]}$ However, the ACCI prognostic score had never been prospectively validated in pelvic cancer surgeries.

CCI has been validated in ovarian cancer by using Danish national database in two studies. ${ }^{[14,15]}$ However, both studies were retrospective in nature, and thus the capture of comorbidities and adverse events both are likely to be inadequate. Similar validation of ACCI in a retrospective setting

Table 3: Details of surgical procedure with length of hospitalization

\begin{tabular}{|c|c|c|c|}
\hline Variable & ACI $<6(n=55)$ & ACI $\geq 6(n=11)$ & Total $(n=66)$ \\
\hline Abdominopelvic resection & $3(5.5)$ & $1(9.1)$ & $4(6.1)$ \\
\hline Anterior resection/low anterior resection/hemicolectomy & $8(14.7)$ & 0 & $8(12.1)$ \\
\hline Anterior resection/low anterior resection/hemicolectomy with multiorgan resection & $2(3.6)$ & 0 & $2(3.0)$ \\
\hline Interval cytoreduction & $9(16.3)$ & $4(36.4)$ & $13(19.7)$ \\
\hline Interval cytoreduction with multiorgan resection & $3(5.5)$ & $4(36.4)$ & $7(10.6)$ \\
\hline Primary cytoreduction & $7(12.7)$ & 0 & $7(10.6)$ \\
\hline Staging laparotomy & $14(25.4)$ & $1(9.1)$ & $15(22.7)$ \\
\hline Radical abdominal hysterectomy & $7(12.7)$ & 0 & $7(10.6)$ \\
\hline Total pelvic exenteration & 0 & $1(9.1)$ & $1(1.6)$ \\
\hline Cystourethrectomy/cystoprostatectomy & $2(3.6)$ & 0 & $2(3.0)$ \\
\hline Lymph node dissection & $50(90.9)$ & $10(90.9)$ & $60(90.9)$ \\
\hline Reexploration & $3(5.4)$ & - & $3(4.5)$ \\
\hline Mean postoperative admission period days & 7.7 (range $3-25$ ) & 9.4 (range 4-18) & 8.0 (range $3-25$ ) \\
\hline
\end{tabular}

ACI=Age-adjusted Charlson comorbidity index

Table 4: Adverse event details

\begin{tabular}{lcccc} 
& Grade 1 (\%) & Grade 2 (\%) & Grade 3 (\%) & Grade 4 \\
\hline Wound infection & $1(1.4)$ & $1(1.4)$ & $4(5.6)$ & - \\
Anastomotic leak & $1(1.4)$ & - & $2(2.8)$ & - \\
Burst abdomen & $1(1.4)$ & - & $2(2.8)$ & - \\
Atrial fibrillation & - & - & $1(1.4)$ & - \\
Acute coronary syndrome & - & $1(1.4)$ & $2(2.8)$ & - \\
Pleural effusion & $1(1.4)$ & - & $2(2.8)$ & - \\
Pulmonary edema & - & - & $2(2.8)$ & - \\
Bed sore & - & $1(1.4)$ & - & - \\
Sepsis & $1(1.4)$ & $1(1.4)$ & - & - \\
Hypontremia & $1(1.4)$ & $15(21.1)$ & - & - \\
Hypokalemia & $15(21.1)$ & - & - & - \\
Hyperkalemia & $3(4.2)$ & - & - & - \\
Hypomagnesemia & $1(1.4)$ & $22(31.0)$ & $4(5.6)$ & - \\
Anemia & $14(19.7)$ & $4(5.6)$ & - & - \\
Urinary tract infection & $6(8.5)$ & - & - & - \\
Deep venous thrombosis & $1(1.4)$ & - & - \\
\hline
\end{tabular}


Table 5: Relation between age-adjusted Charlson comorbidity index and Grade 3-5 adverse events

\begin{tabular}{|c|c|c|c|}
\hline \multirow[t]{2}{*}{ Variable } & \multicolumn{2}{|c|}{ Any grade 3-5 adverse event } & \multirow[t]{2}{*}{ Total } \\
\hline & Yes $(\%)$ & No $(\%)$ & \\
\hline \multicolumn{4}{|l|}{ ACCI score } \\
\hline ACCI $<6$ & $6(10.9)$ & $49(89.1)$ & 55 \\
\hline $\mathrm{ACCI} \geq 6$ & $5(45.5)$ & $6(54.5)$ & 11 \\
\hline Total & 11 & 55 & Grand total $=66$ \\
\hline \multicolumn{4}{|c|}{ ACCI=Age-adjusted Charlson comorbidity index } \\
\hline \multicolumn{4}{|c|}{$\begin{array}{l}\text { Table 6: Relation between Charlson comorbidity index } \\
\text { and Grade } 3-5 \text { adverse events }\end{array}$} \\
\hline \multirow[t]{2}{*}{ Variable } & \multicolumn{2}{|c|}{ Any grade 3-5 adverse event } & \multirow[t]{2}{*}{ Total } \\
\hline & Yes $(\%)$ & No $(\%)$ & \\
\hline \multicolumn{4}{|l|}{ CCI score } \\
\hline CCI $<4$ & $6(11.1)$ & $48(88.9)$ & 54 \\
\hline $\mathrm{CCI} \geq 4$ & $5(41.7)$ & $7(58.3)$ & 12 \\
\hline Total & 11 & 55 & Grand total $=66$ \\
\hline
\end{tabular}

CCI=Charlson comorbidity index

Table 7: Relation between age-adjusted Charlson comorbidity index and Grade 3-5 adverse events

\begin{tabular}{lccc}
\hline & OR & 95\% CI of OR & $P$ \\
\hline ACI score & 4.808 & $0.86-27.0$ & 0.073 \\
Tumor site & 2.538 & $0.45-14.4$ & 0.293 \\
Previous treatment & 4.425 & $0.75-25.6$ & 0.100 \\
\hline
\end{tabular}

$\mathrm{ACI}=$ Age-adjusted Charlson comorbidity index, $\mathrm{OR}=$ Odds ratio, $\mathrm{CI}=$ Confidence interval

was done by Suidan et al. ${ }^{[8]}$ Our study on the other hand had noted all the medical and surgical comorbidities. A list of 25 adverse events both surgical and medical combined were specifically identified and patients were evaluated for same. Morbidity and mortality related to surgical procedures in cancer patients are a source of concern for treating surgeons. Surgical decisions were frequently influenced by patient's age and comorbidity. These decisions were on the basis of subjective assessment of treating physician probably as CCI or ACCI were not validated in the author's country. Post these results, now ACCI needs to be routinely used in clinical practice.

The study is not without its limitations. The study had to be stopped due to slow recruitment. However, the revised sample was powered to show an estimated sensitivity of $85 \%$ with a marginal error rate of 10\%. Approximately 15-20 min were required per patient for ACCI scoring. The devotion of this time in busy outpatient departments of low-middle income countries might be difficult. However, devotion of this time would help surgeons inappropriate resource utilization. A high CCI or ACCI identify patients in whom adverse events are likely and would identify patients in whom the higher cost of treatment would be incurred in future. The study identified that ACCI predicts for development of grade 3-5 adverse events, but the study does not provide an algorithm for surgical workup and interventions in these patients.

\section{Conclusion}

CCI and ACCI can predict for postsurgical adverse events. It has a high negative predictive value for nonoccurrence of adverse events.

\section{Acknowledgment}

The authors would like to thank all staff of MCC.

\section{Financial support and sponsorship}

Nil.

\section{Conflicts of interest}

There are no conflicts of interest.

\section{References}

1. Yeole BB, Kurkure AP, Koyande SS. Geriatric cancers in India: An epidemiological and demographic overview. Asian Pac J Cancer Prev 2008;9:271-4.

2. Read WL, Tierney RM, Page NC, Costas I, Govindan R, Spitznagel EL, et al. Differential prognostic impact of comorbidity. J Clin Oncol 2004;22:3099-103.

3. Patil VM, Chakraborty S, Dessai S, Kumar SS, Ratheesan K, Bindu T, et al. Patterns of care in geriatric cancer patients - An audit from a rural based hospital cancer registry in Kerala. Indian J Cancer 2015;52:157-61.

4. Edwards BK, Noone AM, Mariotto AB, Simard EP, Boscoe FP, Henley SJ, et al. Annual report to the nation on the status of cancer, 1975-2010, featuring prevalence of comorbidity and impact on survival among persons with lung, colorectal, breast, or prostate cancer. Cancer 2014;120:1290-314.

5. Jiao YS, Gong TT, Wang YL, Wu OJ. Comorbidity and survival among women with ovarian cancer: Evidence from prospective studies. Sci Rep 2015;5:11720.

6. Wells CK, Stoller JK, Feinstein AR, Horwitz RI. Comorbid and clinical determinants of prognosis in endometrial cancer. Arch Intern Med 1984; 144:2004-9.

7. Charlson M, Szatrowski TP, Peterson J, Gold J. Validation of a combined comorbidity index. J Clin Epidemiol 1994;47:1245-51.

8. Suidan RS, Leitao MM Jr., Zivanovic O, Gardner GJ, Long Roche KC, Sonoda $\mathrm{Y}$, et al. Predictive value of the Age-Adjusted Charlson Comorbidity index on perioperative complications and survival in patients undergoing primary Debulking surgery for advanced epithelial ovarian cancer. Gynecol Oncol 2015;138:246-51.

9. Hajian-Tilaki K. Sample size estimation in diagnostic test studies of biomedical informatics. J Biomed Inform 2014;48:193-204.

10. Lee JY, Lee DH, Cho NH, Rha KH, Choi YD, Hong SJ, et al. Charlson comorbidity index is an important prognostic factor for long-term survival outcomes in Korean men with prostate cancer after radical prostatectomy. Yonsei Med J 2014;55:316-23.

11. Seo HJ, Yoon SJ, Lee SI, Lee KS, Yun YH, Kim EJ, et al. A comparison of the Charlson comorbidity index derived from medical records and claims data from patients undergoing lung cancer surgery in Korea: A population-based investigation. BMC Health Serv Res 2010; 10:236.

12. Chang CM, Yin WY, Wei CK, Wu CC, Su YC, Yu CH, et al. Adjusted age-adjusted Charlson comorbidity index score as a risk measure of perioperative mortality before cancer surgery. PLoS One 2016;11:e0148076.

13. Hsieh MC, Thompson T, Wu XC, Styles T, O'Flarity MB, Morris CR, et al. The effect of comorbidity on the use of adjuvant chemotherapy and type of regimen for curatively resected stage III colon cancer patients. Cancer Med 2016;5:871-80.

14. Tetsche MS, Dethlefsen C, Pedersen L, Sorensen HT, Norgaard M. The impact of comorbidity and stage on ovarian cancer mortality: A nationwide Danish cohort study. BMC Cancer 2008;8:31.

15. Grann AF, Thomsen RW, Jacobsen JB, Nørgaard M, Blaakær J, Søgaard $\mathrm{M}$, et al. Comorbidity and survival of Danish ovarian cancer patients from 2000-2011: A population-based cohort study. Clin Epidemiol 2013;5:57-63. 